\title{
Images of the month: Demonstrative oral mucosal sarcoidosis in a patient with pulmonary disease
}

\author{
Authors: Duygu Gulseren ${ }^{A}$ and Gonca Elçin ${ }^{B}$
}

Sarcoidosis is a multisystem granulomatous disease that preferentially affects the lungs and intrathoracic lymph nodes. Oral involvement is quite rare and usually appears in patients with known systemic sarcoidosis but it may also be the initial finding of a systemic disease. Herein, we report a case of asyptomatic pulmonary sarcoidosis with cutaneous and oral involvement. Recognising oral sarcoidosis may be difficult but it is important not to miss an asymptomatic pulmonary sarcoidosis. Patients with oral lesions of sarcoidosis should be screened for systemic disease.

KEYWORDS: Dermatology, sarcoidosis

DOI: $10.7861 /$ clinmed.2020-0257

\section{Introduction}

Sarcoidosis is a multisystem granulomatous disease that preferentially affects the lungs and intrathoracic lymph nodes, less frequently the skin, eyes, salivary glands, spleen, nervous system or the heart. ${ }^{1}$ Oral involvement is quite rare and usually appears in patients with known systemic sarcoidosis but it may also be the initial finding of a systemic disease. ${ }^{2}$ We report a case of pulmonary sarcoidosis with cutaneous and oral involvement and we would like to emphasise the importance of recognising the oral presentation of sarcoidosis to establish the definitive diagnosis of systemic sarcoidosis in otherwise asymptomatic patients.

\section{Case presentation}

A 31-year-old man was referred to our dermatology clinic for yellow to pink colored lesions on his nose and mouth. The lesions had appeared 3 months previously. The patient declared that the lesion on his nose started like an acne pimple and his mouth lesion looked similar to oral aphthae but they did not resolve and slowly grew. He did not report any systemic or dermatological symptoms related with his lesions. His medical history revealed that he suffered from gout disease and it resolved with oral allopurinol therapy. On dermatologic examination, there was a yellowish

Authors: A assistant professor, Hacettepe University, Ankara,

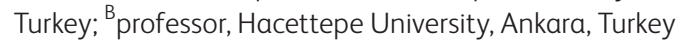

erythematous, telangiectatic, infiltrated plaque with a diameter of $13 \times 10 \mathrm{~mm}$ on his nasal tip, close to his columella. Intraorally, there was an indurated, non-tender, firm, scar-like plaque extending from the lower labial mucosa to the frenulum (Fig 1). The regional lymph nodes were not enlarged clinically. Two skin punch biopsies were taken from his nasal tip and lower labial mucosa. Both biopsies revealed granuloma formation composed of epithelioid histiocytes and multinucleated giant cells with a thin peripheral rim of lymphocyte localised in the dermis and lamina propria. To rule out other granulomatous diseases like cutaneous tuberculosis, the Ziehl-Neelsen stain was performed and it was negative for acid-fast bacilli. The serum angiotensin converting enzyme (ACE) level was higher than normal at 76 IU/L (normal range 8.0-52.0).
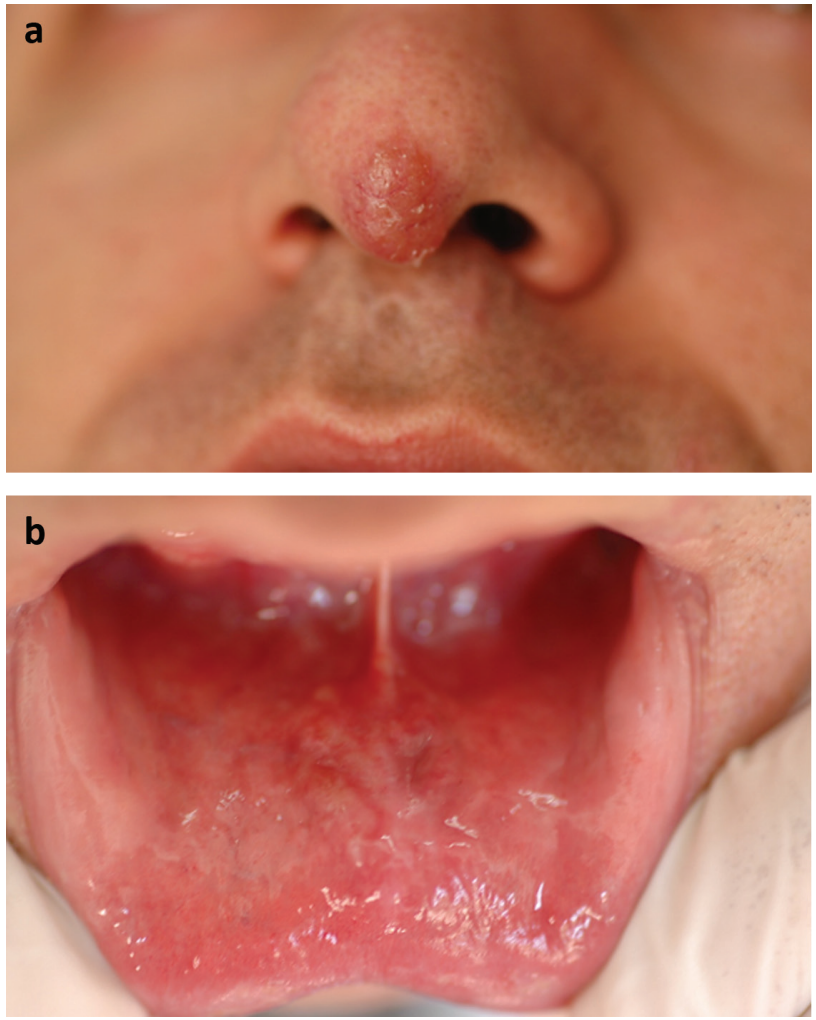

Fig 1. a) Fine scaly, erythematous, infiltrated plaque on the nasal tip. b) Indurated, scar-like plaque in the midline of the lower lip. 
Computed tomography demonstrated numerous bilateral hilar and mediastinal lymphadenopathy and there was a fibrotic band on the anterobasal segment of the right lung. Based on these clinical, laboratory, histopathological and radiological findings, the diagnosis of pulmonary sarcoidosis with cutaneous and oral involvement was made. After starting oral hydroxychloroquine therapy, cutaneous and oral lesions disappeared within 6 months.

\section{Discussion}

Involvement of the skin is the most common extrathoracic manifestation of sarcoidosis and cutaneous lesions may present in $20-35 \%$ of cases. ${ }^{3,4}$ Cutaneous lesions are commonly characterised by papules, plaques (including angiolupoid and lupus pernio) and nodules; rarer forms include ulcerated, lichenoid, atrophic, psoriasiform, ichthyosiform and vitiligolike presentations. Although dermatologists are familiar with cutaneous lesions due to the relatively frequent involvement, oral lesions of sarcoidosis are rarely encountered. Suresh et al reviewed 68 previously published cases of oral involvement in sarcoidosis with histopathological confirmation and they reported that the soft tissues of the oral cavity were affected in 47 cases and the jaw bones in 21 cases. ${ }^{5}$ Among the soft tissues, buccal mucosa was the commonest site affected with 13 cases followed by gingiva (10), lips (six), floor of the mouth/sublingual gland (five), tongue (five), palate (three), submandibular gland (two) and multiple oral site involvement (two). Clinical presentations of the lesions have not been properly documented, they were imprecisely described such as localised swelling or nodules in 34 cases, ulcers (four), swelling with multiple ulcers (two), gingivitis (three), gingival hyperplasia (three) and gingival recession (one). Oral lesions were the initial finding of the systemic sarcoidosis in 24 cases. Nico et al compared oral mucosal lesions of sarcoidosis with their cutaneous counterparts in the same patients and they found very similar presentation in morphology, shape and size to their cutaneous lesions. ${ }^{4}$ Also, our patient was a very demonstrative case for the classical morphology, localisation, shape and size of both the cutaneous and oral sarcoidosis.

\section{Conclusion}

In conclusion, recognising oral sarcoidosis may be difficult but it is important not to miss an asymptomatic pulmonary sarcoidosis. Patients with oral lesions of sarcoidosis should be screened for systemic disease.

\section{References}

1 Motswaledi MH, Khammissa RA, Jadwat Y, Lemmer ], Feller L. Oral sarcoidosis: a case report and review of the literature. Aust Dent J 2014:59:389-94.

2 Kasamatsu A, Kanazawa H, Watanabe T, Matsuzaki O. Oral sarcoidosis: report of a case and review of literature. J Oral Maxillofac Surg 2007;65:1256-9.

3 Ungprasert P, Ryu JH, Matteson EL. Clinical manifestations, diagnosis, and treatment of sarcoidosis. Mayo Clin Proc Innov Qual Outcomes 2019:3:358-75.

4 Nico MM, Guimarães AL, Correa PY, Lourenço SV. Oral mucosal lesions in sarcoidosis: comparison with cutaneous lesions. Acta Derm Venereol 2016;96:392-3.

5 Suresh L, Radfar L. Oral sarcoidosis: a review of literature. Oral Dis 2005;11:138-45.

Address for correspondence: Dr Duygu Gülseren, Department of Dermatology, School of Medicine, Hacettepe University, 06230 Ankara, Turkey.

Email: duygu_gulsrn@hotmail.com 